\title{
An in vitro Evaluation of Antibacterial Activity of Curcumin against Aggregatibacter actinomycetemcomitans
}

\author{
${ }^{1}$ Abdulpur Mallikarjun Savita, ${ }^{2}$ Charu Dawra, ${ }^{3}$ Kishore Bhat
}

\begin{abstract}
Introduction: Development of bacterial resistance to the available antibiotics and increasing popularity of traditional medicine has led researchers to investigate the antibacterial compounds in plants. In this context, curcumin has been shown to have a range of beneficial properties, like antimicrobial and anti-inflammatory properties, which might be favorable for the treatment of inflammatory diseases like periodontitis.
\end{abstract}

Aims and objectives: The primary objective of this study was to evaluate the antibacterial potential of curcumin against Aggregatibacter actinomycetemcomitans (ATCC 29523).

Materials and methods: The bacterial strains of $A$. actinomycetemcomitans from the stock were revived and transferred to sterile brain heart infusion $(\mathrm{BHI})$ broth. Minimum inhibitory concentration (MIC) was determined by serial broth dilution of curcumin. The last tube with clear supernatant was considered to be without any growth and taken as MIC value.

Results: The mean MIC value of curcumin against $A$. actinomycetemcomitans (ATCC 29523) was reported to be $0.2 \mu \mathrm{g} / \mathrm{ml}$.

Conclusion: Thus, it can be concluded that the pleiotropic activities of curcumin derived from its complex chemistry and its ability to influence and control the multiple signaling pathways, makes it a suitable and safer option for the treatment of periodontal diseases.

Keywords: Antibacterial, Aggregatibacter actinomycetemcomitans, Curcumin, MIC.

How to cite this article: Savita AM, Dawra C, Bhat K. An in vitro Evaluation of Antibacterial Activity of Curcumin against Aggregatibacter actinomycetemcomitans. World J Dent 2015;6(1):16-19.

\section{Source of support: Nil}

Conflict of interest: None

\section{INTRODUCTION}

Periodontal disease is a chronic inflammatory disease of the supporting tissues of the teeth, triggered by the host's immune response to microbial plaque. This results in the

\footnotetext{
${ }^{1,3}$ Professor and Head, ${ }^{2}$ Postgraduate Student

1,2Department of Periodontics, Dayananda Sagar College of Dental Sciences, Bengaluru, Karnataka, India

${ }^{3}$ Department of Microbiology, Maratha Mandal's NGH Institute of Dental Sciences and Research Centre, Belgaum, Karnataka, India

Corresponding Author: Charu Dawra, Postgraduate Student Department of Periodontics, Dayananda Sagar College of Dental Sciences, Shavige Malleshwara Hills, Kumaraswamy Layout Bengaluru-560078, Karnataka, India, Phone: +91-9900408428, e-mail: charu.dawra@gmail.com
}

progressive loss of tooth-supporting tissues, which ultimately can lead to premature tooth loss. Gingivitis and periodontitis, which are the two most common forms of periodontal diseases, are caused by the bacteria present in the plaque biofilm. This biofilm community is initially formed through bacterial interactions with the tooth and then through physical and physiologic interactions among different species within the microbial mass. ${ }^{1}$ Furthermore, the bacteria found in the plaque biofilm are strongly influenced by external environmental factors that may be host mediated.

Various microorganisms are known to be associated with periodontitis, such as Porphyromonas gingivalis, Prevotella intermedia, Aggregatibacter actinomycetemcomitans, Fusobacterium nucleatum, etc. ${ }^{2}$ However, elevated proportions of some predominant subgingival microbial species like $P$. gingivalis and A. actinomycetemcomitans, have been associated with destructive periodontal disease activity. ${ }^{3}$

Thus, an essential component of periodontal therapy is to suppress the pathogenic microflora of the pocket, which may be accomplished by either non surgical or surgical means. ${ }^{4}$ Nonsurgical approach in the form of scaling and root planning has proven to be successful in immediately reducing the microbial load but it sometimes fails to eliminate the bacteria in inaccessible areas, leading to recurrence of disease.

Hence, the use of antimicrobial agents, both systemic and topical, has eventually increased because of the realization that periodontal disease is not only an overgrowth of bacteria, but also a shift in bacterial species. ${ }^{5}$

Systemic administration of antimicrobial drugs has been useful in treating periodontal pockets. However, it involves consumption of a relatively high dose with repeated intake over a prolonged period of time to achieve the required inhibitory concentrations in the sulcular fluid. This increases the possibility of development of resistance, alteration of commensal flora and increased potential for adverse effects, such as allergic/anaphylactic reactions, gastric disturbances, superinfection, nausea, vomiting, etc. ${ }^{6}$

Local administration of these antimicrobial drugs provides answers to problems of systemic administration with no systemic side-effects. At the same time, this approach again relies on the ability to control and prolong the release rate of the therapeutic agent from the device. 
Owing to the clinical drawbacks of synthetic agents (both systemic and topical), there was an imminent need of an alternative medicine that could provide a product already enmeshed within the traditional Indian setup and is also safe and economical.

In this context, Curcuma longa, a medicinal plant that botanically is related to Zingiberaceae family, was the need of the hour. Curcuma longa, commonly known as 'turmeric', is widely used as a spice and coloring agent, and is well known for its medicinal properties. ${ }^{8}$ Components of turmeric are called curcuminoids, which mainly include curcumin (diferuloylmethane), demethoxycurcumin and bisdemethoxycurcumin. ${ }^{9}$ Among the three components, curcumin is the most important fraction which is responsible for the biological activities of turmeric..$^{10}$ It has been shown that curcumin has a wide spectrum of biological actions, such as antibacterial, anti-inflammatory, antioxidant, antidiabetic, anticancer, antiallergic and many more. ${ }^{11}$

Recent research has also proven that periodontal breakdown is caused by interaction of bacteria with the host immune response with the help inflammatory mediators and free radicals. Therefore, the antibacterial, anti-inflammatory and antioxidant properties of curcumin are desirable assets which might validate its use in the treatment of periodontitis.

A. actinomycetemcomitans is a Gram-negative capnophilic bacterium. Due to the tissue invasion property of this bacterium, it is regarded as a pathogen associated with one forms of periodontitis-localized aggressive periodontitis. ${ }^{12}$ This bacterium is also associated with systemic infections that can result in endocarditis, meningitis and brain abscesses. ${ }^{13}$

Therefore, this study was conducted to evaluate the antibacterial role of curcumin against one of the most tissue invasive periodontal pathogen-A. actinomycetemcomitans.

\section{MATERIALS AND METHODS}

The test agent used in this study was 5\% curcumin (Sami Labs Limited, Peenya, Bengaluru, Karnataka). The rhizomes of Curcuma longa were extracted using acetone as a solvent and the extract was then dissolved with isopropyl alcohol to achieve a final extract ratio of 50:1 to 60:1.

The test organism was A. actinomycetemcomitans (ATCC 29523).

\section{Bacterial Strains and Maintenance Procedure}

The needed bacterial strain from the stock were revived by plating on blood agar medium. After overnight incubation at $37^{\circ} \mathrm{C}$, those were transferred to sterile BHI broth and once again incubated overnight. The growth concentration was adjusted to $5 \times 10^{5}$ organisms $/ \mathrm{ml}$ by using 0.5 McFarland's turbidity standard.

\section{Determination of Minimum Inhibitory Concentration ${ }^{14}$}

Stock solution of the test agent (curcumin) was made up in DMSO (dimethylsulfoxide) to ensure complete solubilization. Nine dilutions of each drug have to be done with BHI for minimum inhibitory concentration (MIC). In the initial tube, $20 \mu \mathrm{l}$ of drug was added to $380 \mu \mathrm{l}$ of BHI broth. For dilutions, $200 \mu \mathrm{l}$ of BHI broth was added into the next nine tubes separately. Then from the initial tube, $200 \mu \mathrm{l}$ was transferred to the first tube containing $200 \mu \mathrm{l}$ of BHI broth. This was considered as 10-1 dilution. From 10-1 diluted tube, $200 \mu 1$ was transferred to second tube to make 10-2 dilution. The serial dilution was repeated upto 10-9 dilution for each drug. By following this serial dilution, the concentrations of the aqueous extract achieved was the following-100, 50, 25, 12.5, 6.25, 3.12, $1.6,0.8,0.4,0.2 \mu \mathrm{g} / \mathrm{ml}$ respectively.

To each of the 10 such prepared MIC tubes with varying concentrations, $200 \mu \mathrm{l}$ of the earlier prepared strain of organism was added such that the final volume per tube as $400 \mu l$. The tubes were then incubated for 24 hours at $37^{\circ} \mathrm{C}$.

After the incubation, the MIC values were determined by visual inspection of the tubes. In each series of tubes, the last tube with clear supernatant was considered to be without any growth and taken as MIC value. Turbidity in the MIC tube indicated growth of the bacteria implying that the bacteria are resistant to curcumin (Fig. 1).

\section{RESULTS}

The serial dilutions of curcumin extract with turbidity response of A. actinomycetemcomitans is summarized as follows (Table 1).

Thus, curcumin has the ability to exert antibacterial activity against $A$. actinomycetemcomitans at a minimum inhibitory concentration of $0.2 \mu \mathrm{g} / \mathrm{ml}$.

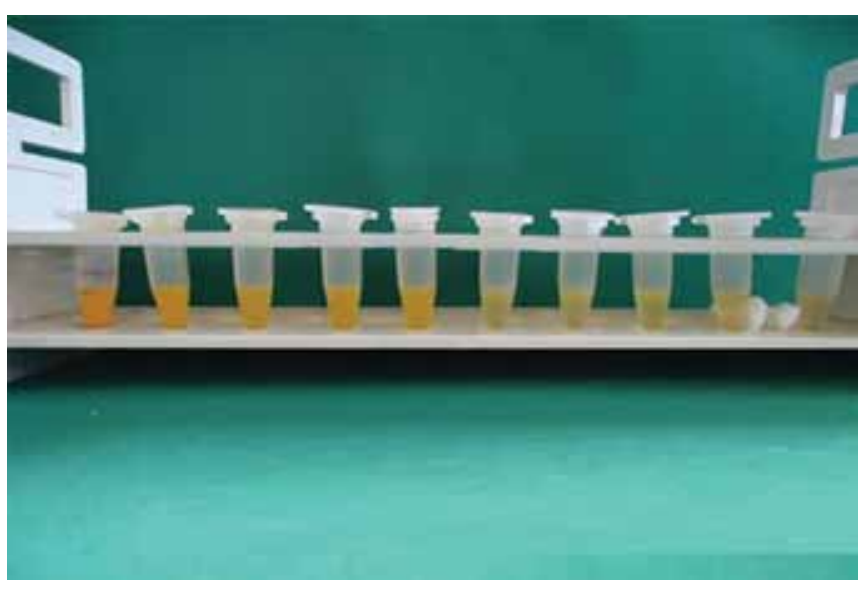

Fig. 1: Turbidity response of $A$. actinomycetemcomitans against serial dilutions of curcumin to check for MIC 
Table 1: Serial dilutions of curcumin extract with turbidity response of $A$. actinomycetemcomitans

\begin{tabular}{lllllllllllll}
\hline S. & Curcumin \\
no. & extract & 100 & 50 & 25 & 12.5 & 6.25 & 3.12 & 1.6 & 0.8 & 0.4 & 0.2 \\
\hline 1 & Aa & S & S & S & S & S & S & S & S & S & S \\
\hline
\end{tabular}

Note: Aa: A. actinomycetemcomitans; $\mathrm{S}$ : Susceptible

\section{DISCUSSION}

Despite the availability of a wide range of antimicrobial agents for clinical use, development of new antimicrobial agents remains important and many studies have been aiming at the discovery and development of new antimicrobial agents. ${ }^{15}$

The combination of microorganisms and inflammatory response are the cause of many diseases, including periodontitis, for which compounds having a dual antiinflammatory and antimicrobial activity may be desirable therapeutic agents. ${ }^{16}$ Globally, plant extracts are employed for their antibacterial, antifungal and antiviral activities. It is known that more than 400,000 species of tropical flowering plants have medicinal properties and this has made traditional medicine cheaper than modern medicine. ${ }^{17}$

In this study, it was thus suggested that curcumin has the ability to exert antibacterial activity against one of the most tissue invasive periodontal pathogenA. actinomycetemcomitans, at a minimum inhibitory concentration of $0.2 \mu \mathrm{g} / \mathrm{ml}$.

Thus, the results of this study corroborate with the findings of study by Mandroli et al, which was done to evaluate the ability of curcumin to exert antibacterial activity against common endodontic bacteria like Streptococcus mutans, Actinomyces viscosus, Lactobacillus casei, P. gingivalis, Prevotella intermedia and Enterococcus faecalis. ${ }^{18}$ It was, hence, suggested that curcumin has the ability to kill several Gram-positive and Gram-negative bacteria.

A study by Rai et al suggested that curcumin may inhibit bacterial cell proliferation by inhibiting the assembly dynamics of FtsZ (a bacterial protofilament), which polymerizes to form a Z-ring at the midcell that orchesterates bacterial cell division. The assembly and stability of FtsZ protofilaments have been shown to play critical roles in bacterial cytokinesis. Thus, FtsZ may be considered as an important antibacterial drug target. It was suggested that curcumin strongly inhibited the formation of the cytokinetic Z-ring, which would prove lethal to bacteria, thus, accounting for curcumin's antibacterial activity. ${ }^{19}$

Another reason which might be suggested for the antibacterial potential of curcumin is that curcumin is a polyphenolic compound, also having substantial antibiofilm activity. ${ }^{20}$ It inhibits the production of biofilm and disperses the biofilm made by many microorganisms. Owing to the plaque biofilm acting as the main etiological factor in the periodontal diseases, curcumin's antibiofilm activity would also account for its antibacterial activity against periodontal pathogens.

Apart from the antibacterial property, curcumin is a molecule known to demonstrate antifungal activity ${ }^{21}$ and antiviral activity as well. ${ }^{22}$

Further, curcumin has long been touted as a powerful anti-inflammatory and immunomodulatory agent. ${ }^{23}$ Curcumin has been shown to regulate numerous transcription factors, cytokines, protein kinases, adhesion molecules, redox status and enzymes that have been linked to inflammation.

The anti-inflammatory activity of curcumin was first reported in $1971 .{ }^{24}$ It reduces inflammation by effectively inhibiting transcriptional and translational expression of proinflammatory cytokines like IL- 6 and TNF- $\alpha$. Curcumin also demonstrated dose-dependent attenuation of NF- $\kappa \beta$ activation in the gingival tissue of rats suffering from experimental periodontal disease. It, further, reduced the inflammatory infiltrate, increased the collagen content, and increased fibroblastic cell numbers in gingival tissues. ${ }^{25}$

Because of the increasing evidence that the pathogenesis of type 2 diabetes mellitus (T2DM) and obesity is connected to inflammation, curcumin has emerged as a potential drug of interest for diabetic and obesity pharmacology. ${ }^{26}$

Of all the possible clinical applications of curcumin, none has garnered more interest and investment of research resources and manpower than the potential use of curcumin as a cancer chemopreventive and chemotherapeutic agent. ${ }^{27}$ Curcuminoids possess anticarcinogenic property due to their oxygen radical-scavenging property. ${ }^{28}$ This interest and this voluminous amount of investigations continue unabated.

Despite extensive research and development, poor oral bioavailability of curcumin remains one of the major concerns for its use as a therapeutic agent. This poor oral bioavailability of curcumin can be attributed to its poor absorption, high rate of metabolism in the intestines and rapid systemic elimination from the body. Thus, attempts have been made to improve curcumin's bioavailability by use of adjuvants that can block the metabolic pathway of curcumin. This has been achieved through encapsulation in liposomes, biodegradable microspheres, cyclodextrin, and hydrogels. Also, various controlled delivery forms, such as polymeric micro/nanospheres, liposomes, micelles, parenteral emulsion, and prodrugs have been investigated to increase its solubility. 


\section{CONCLUSION}

Due to multiple therapeutic properties and pleiotropic roles, curcumin can be considered a safe, nontoxic and effective alternative for the treatment of inflammatory conditions like periodontitis. However, further research is warranted to determine the optimal dosage, bioavailabilty and bioefficacy of curcumin-based drugs. As the number of research studies on therapeutic effects of curcumin keeps on increasing, it is evident that curcumin truly holds a promising future in therapeutic applications including dentistry.

\section{ACKNOWLEDGMENTS}

SAMI LABS LIMITED, Peenya, Bengaluru, Karnataka.

\section{REFERENCES}

1. Kuramitsu HK, He X, Lux R, Anderson MH, Shi W. Interspecies interactions within oral mircobial communities. Microbiol Mol Biol Rev 2007;71(4):653-670.

2. van Winkelhoff AJ, Loos BG, van der Reijden WA, van der Velden U. Porphyromonas gingivalis, Bacteroides forsythus and other putative periodontal pathogen in subjects with and without periodontal destruction. J Clin Periodontol 2002;29(11):1023-1028.

3. Kesic L, Milasin J, Igic M, Obradovic R. Microbial etiology of periodontal disease-Mini Review. Facta Universitatis 2008;15(1):1-6.

4. Ryan ME. Nonsurgical Approaches for the treatment of periodontal diseases. Dent Clin N Am 2005;49(3):611-636.

5. Nayyar N, Dodwad V, Arora K. Comparative evaluation of $1 \%$ curcumin solution and $0.2 \%$ chlorhexidine irrigation as an adjunct to scaling and root planing in management of chronic periodontitis: A clinico-microbiological study. JPBMS 2012;14:1-6.

6. Puri K, Dodwad V, Bhat K, Puri N. Effect of controlled-release Periochip ${ }^{\mathrm{TM}}$ on clinical and microbiological parameters in patients of chronic periodontitis. J Indian Soc Periodontol 2013;17(5):605-611.

7. Hayakawa H, Minaniya Y, Ito K, Yamamoto Y, Fukuda T. Difference of curcumin content in Curcuma longa L. (Zingiberaceae) caused by hybridization with other curcuma species. AJPS 2011;2:111-119.

8. Luthra PM, Singh R, Chandra R. Therapeutic uses of Curcuma longa (Turmeric). Indian J Clin Biochem 2001;16(2):153-160.

9. Nagpal M, Sood S. Role of curcumin in systemic and oral health: An overview. J Nat Sci Biol Med 2013;4(1):3-7.

10. Naz S, Jabeen S, Ilyas S, Manzoor F, Aslam F, Ali A. Antibacterial activity of Curcuma longa varieties against different strains of bacteria. Pak J Bot 2010;42:455-462.

11. Anand P, Thomas SG, Kunnumakkara AB, Sundaram C, Harikumar KB, Sung B et al. Biological activities of curcumin and its analogues (Congeners) made by man and Mother Nature. Biochem Pharmacol 2008;76(11):1590-1611.

12. Kuboniwa M, Tribble GD, Hendrickson EL, Amano A, Lamont RJ, Hackett M. Insights into the virulence of oral biofilms: discoveries from proteomics. Expert Rev Proteomics 2012;9(3):311-323.

13. Barak O, Dashper SG, Catmull DV, Adams GG, Sela MN, Machtei EE et al. Antibiotic susceptibility of Aggregatibacter actinomycetemcomitans JP2 in a biofilm. J Oral Microbiol 2013;5:203-220.

14. Antimicrobial susceptibility testing protocols. Schwalve, Moore and Goodwin, Crc Press; 2007.

15. Mukhopadhyay A, Peterson RT. Fishing for new antimicrobials. Curr Opin Chem Biol 2006;10(4):327-333.

16. Ishikawa I. Host responses in periodontal diseases: a preview. Periodontol 2000, 2007;43:9-13.

17. Odugbemi T. Medicinal plants as antimicrobials In: Outline and pictures of medicinal plants from Nigeria. University of Lagos Press; p. 53-64.

18. Mandroli PS, Bhat K. An in vitro evaluation of antibacterial activity of curcumin against common endodontic bacteria. J App Pharm Sci 2013;3(10):106-108.

19. Rai D, Singh JK, Roy N, Panda D. Curcumin inhibits FtsZ assembly: an attractive mechanism for its antibacterial activity. Biochem J 2008;410(1):147-155.

20. Chusri S, Sompetch K, Mukdee S, Jansrisewangwong S, Srichai T, Maneenoon K, et al. Inhibition of Staphylococcus epidermidis biofilm formation by traditional Thai herbal recipes used for wound treatment. Evid Based Complement Alternat Med 2012;2012:159797.

21. Neelofar K, Shreaz S, Rimple B, Muralidhar S, Nikhat M, Khan LA. Curcumin as a promising anticandidal of clinical interest. Can J Microbiol 2011;57(3):204-210.

22. Zandi K, Ramedani E, Mohammadi K, Tajbakhsh S, Deilami I, Rastian Z, et al. Evaluation of antiviral activities of curcumin derivatives against HSV-1 in Vero cell line. Nat Prod Commun 2010;5(12):1935-1938.

23. Madan B, Ghosh B. Diferuloylmethane inhibits neutrophil infiltration and improves survival of mice in high-dose endotoxin shock. Shock 2003;19:91-96.

24. Srimal RC, Khanna KM, Dhawan BN. A preliminary report on anti inflammatory activity of curcumin. Ind J Pharmacol 1971;3:10

25. Guimaraes MR, Coimbra LS, de Aquino SG, Spolidorio LC, Kirkwood KL, Rossa C Jr. Potent anti-inflammatory effects of systemically administered curcumin modulate periodontal disease in vivo. J Periodontal Res 2011;46(2):269-279.

26. Aggarwal B. Targeting inflammation-induced obesity and metabolic diseases by curcumin and other nutraceuticals. Annu Rev Nutr 2010;30:173-199.

27. Varinska L, Mirossay L, Mojzisova G, Mojzis J. Antiangogenic effect of selected phytochemicals. Pharmazie 2010;65(1):57-63.

28. Kohli K, Ali J, Ansari MJ, Raheman Z. Curcumin: a natural anti-inflammatory agent. Ind J Pharmacol 2005;37:141-147. 